\title{
Comparison of Kirschner wires and AO cannulated screw internal fixation for displaced lateral humeral condyle fracture in children
}

\author{
Wen Chao Li • Rui Jiang Xu
}

Received: 26 October 2011 / Accepted: 27 November 2011 /Published online: 17 December 2011

(C) Springer-Verlag 2011

\begin{abstract}
Purpose This retrospective study compares Kirschner wires versus 3.5-mm diameter AO cannulated screw internal fixation in treatment for the displaced lateral humeral condyle fractures.

Methods The study included 62 patients (42 boys, 20 girls; mean age 6.93 years; age range two to 14 years) with displaced lateral humeral condyle fractures. All patients were treated by open reduction and Kirschner wires or cannulated screw fixation. The clinical outcomes were evaluated according to the criteria of Hardacre et al. The mean follow-up period was 39.4 months (range 21-95 months). Results There was no statistically significant difference in clinical outcome between these two groups $(P>0.05)$. Five patients $(16.7 \%)$ developed skin infection around K-wires, while no infection occurred in fracture with screws. An obvious lateral prominence occurred in 11 (36.7\%) patients with K-wires and four (12.5\%) patients with screws. Nine $(30 \%)$ patients with $\mathrm{K}$-wires and two $(6.3 \%)$ patients with screws had a lack of $10^{\circ}$ of extension of the elbow compared with the other side.

Conclusion Both K-wires and cannulated screw fixation are effective in treatment for displaced lateral humeral condyle fracture. K-wires can pass through the ossific nucleus of capitulum without damaging it, but a longer period of external fixation and local skin care will be required. The screws can reduce the possibility of lateral prominence and promote the function of elbow by continuously stabilising the fracture, but a second operation is need for screw removal.
\end{abstract}

W. C. Li $\cdot$ R. J. Xu $(\bowtie)$

Department of Paediatric Orthopaedic Surgery, Chinese People's

Liberation Army General Hospital,

Beijing 100853, China

e-mail: xurj.2006@yahoo.com.cn

\section{Introduction}

Lateral humeral condyle fracture, the second most common injury around the elbow, accounts for $10-20 \%$ of all fractures of the elbow in children with a high incidence between two and 14 years [1-5]. The fractures have a high risk for complications including nonunion, malunion, ulnar nerve paresis, hypertrophic scar, avascular necrosis of ossific nucleus and angular deformity [6-9]. Various treatment methods are recommended according to the degree of displacement. It has been generally accepted that lateral humeral condyle fractures with displacement more than $2 \mathrm{~mm}$ should be treated by open reduction and internal fixation [10-17]. Therefore, most of the researchers recommend open reduction and Kirschner wires (K-wires) fixation in the displaced lateral condyle fracture $[10,11,16]$. Although K-wire is the most common metallic implant, a plaster splint or cast is required for a period of immobilisation. Some authors suggest that screw fixation also promotes the union of fracture without significant complications $[12-14,17]$. However, there have been no published reports comparing cannulated screws to K-wires in the displaced lateral condyle fractures.

Here, we retrospectively reviewed patients treated with different internal fixation of $\mathrm{K}$-wires or $3.5-\mathrm{mm}$ diameter AO cannulated screws to evaluate the clinical outcome for the displaced lateral humeral condyle fractures.

\section{Materials and methods}

A retrospective review of 62 patients with displaced lateral condyle fractures with varying degrees of displacement was performed from January 2000 to December 2008. Patients were treated by open reduction and $\mathrm{K}$-wires or $3.5-\mathrm{mm} \mathrm{AO}$ 
cannulated screw fixation. The clinical records and radiographs of patients were retrospectively analysed. Fractures were classified according to the Milch system [18]. All patients underwent anteroposterior and lateral radiographs, and the displacement of the fracture fragment in radiographs was measured and recorded. The fracture gap was measured from lateral metaphyseal cortex of the distal humerus to the lateral cortex of the fracture fragment. The greatest displacement on any single radiograph was considered as the amount of displacement of the fracture (Fig. 1). The demographic data and follow-up information are summarised in Table 1.

Informed consent from all patients and patients' parents or guardians was obtained. A traditional lateral approach to the elbow was performed following general anaesthesia. Care was taken to limit damage to the soft tissue and periosteum. After open reduction of the fracture, K-wires or cannulated screws were used to stabilise the fracture. K-wires or screws were required to pass through the metaphyseal fragment. If there was not enough space in the metaphyseal fragment, K-wires or screws might pass through the ossific nucleus of the capitulum. All K-wires were placed percutaneously into the fractures.

After operation, an above-elbow plaster splint was used to support the fracture fixed with K-wires for five or six weeks. When the fracture united, the percutaneous K-wires were removed at an outpatient clinic without anaesthesia six or eight weeks after the operation. The fracture with screws was supported by an above-elbow brace for one or two weeks. After the brace was removed, the patient was required to do exercises of the elbow. When radiographs showed sufficient evidence of fracture union, screws were routinely removed at eight or 12 weeks in the operating room under general anaesthesia.

The mean follow-up period was 39.4 months (range 2695 months). An independent observer reviewed all records of charts and radiographs in order to ensure the uniformity of analysis. The clinical information in the two groups was recorded including the patient's age at injury, the type of fixation, the period of K-wires or screw fixation, the functional result of the elbow and complications. In addition, radiographic information including the union of fractures, nonunion, malunion or lateral prominence was recorded for the radiographic evaluation. The clinical results were evaluated according to the criteria suggested by Hardacre et al. [19].

\section{Statistical analysis}

Statistical analysis was performed using the Pearson chi-square test. With theoretical samples less than 5, the Fisher's exact test was used. $P<0.05$ was considered statistically significant.

\section{Results}

Of the 62 displaced fractures, 30 patients (average displacement $5.5 \mathrm{~mm}$, range $2-10 \mathrm{~mm}$ ) were treated by open reduction and K-wire fixation (Fig. 2). These fractures were fixed with two K-wires (23 fractures, $76.7 \%$ ) or three K-wires (seven fractures, 23.3\%). K-wires passed through the metaphyseal fragment in 24 patients and the ossific nucleus of capitulum in six patients. Thirty-two patients (average displacement $6.0 \mathrm{~mm}$, range $2-10 \mathrm{~mm}$ ) were treated by open reduction and screw fixation (Fig. 3). Twenty-seven (84.4\%) fractures were stabilised with two screws, and five (15.6\%) fractures with one screw. Screws passed through the metaphyseal fragment in 28 patients and the ossific nucleus of capitulum in four patients.

According to the criteria of Hardare et al., clinical outcomes in the group with K-wires were excellent in 22
Fig. 1 The gap of fracture was measured in the anteroposterior and lateral radiographs from lateral metaphyseal cortex of the distal humerus to the lateral cortex of the fracture fragment. The greatest displacement on any single radiograph was considered as the amount of displacement of the fracture
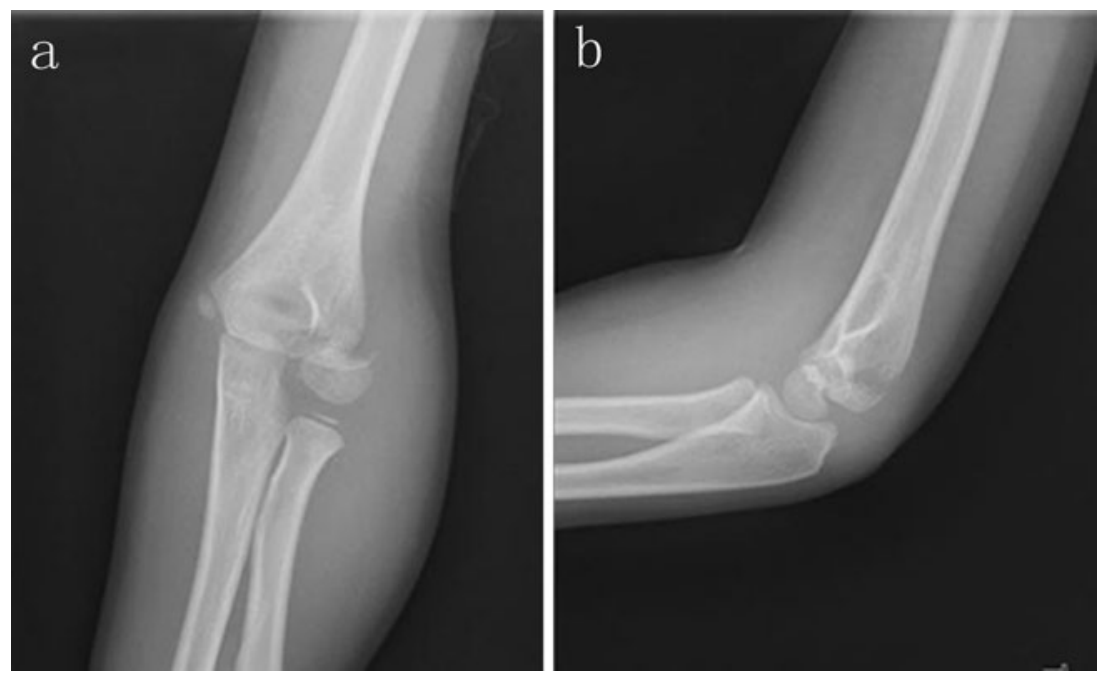
Table 1 Demographic data for displaced fractures fixed with K-wires or cannulated screws

\begin{tabular}{lll}
\hline Parameter & $\begin{array}{l}\text { Fractures with } \\
\text { K-wires }\end{array}$ & $\begin{array}{l}\text { Fractures with } \\
\text { cannulated screws }\end{array}$ \\
\hline Number of patients & 30 & 32 \\
Boys & $24 / 30$ & $18 / 32$ \\
Girls & $6 / 30$ & $14 / 32$ \\
Mean age at injury (years) & 6.83 & 7.02 \\
Right side & $17 / 30$ & $20 / 32$ \\
Left side & $13 / 30$ & $12 / 32$ \\
Milch I & $5 / 30$ & $9 / 32$ \\
Milch II & $25 / 30$ & $23 / 32$ \\
Mean follow-up (months) & 40.1 & 38.8 \\
\hline
\end{tabular}

patients, good in eight patients and none were poor. Clinical outcomes in the other group with screws were excellent in 21 patients, good in 11 patients and poor in none. No statistically significant difference was found in clinical results between $\mathrm{K}$-wires and cannulated screw fixation $(P>0.05)$. No non-union, avascular necrosis of ossific nucleus or clinically significant epiphysial fusion occurred in the two groups.

Five patients (16.7\%) presented skin infection around Kwires. The infections were treated with oral antibiotics and local wound care, and the period of plaster splinting was extended to nine or ten weeks to prevent loosening of the Kwires. Last follow-up radiographs showed all fractures successfully achieved union. In contrast, no infection occurred in the fracture fixed with screws. Seven K-wires in four fractures were found to be loose when they did not pass through the cortex of the opposite side. When the K-wires were removed at ten or 12 weeks, all fractures had achieved union without loss of reduction.

An obvious clinical and radiograph prominence over lateral humeral condyle occurred in 11 (36.7\%) patients with K-wires and four $(12.5 \%)$ patients with screws, but no patients required surgery. The difference in lateral prominence of the two groups was statistically significant $(P<0.05)$.

Seven $(23.3 \%)$ patients with K-wires had a significant difference in their carrying angles, with relative cubitus varus in six patients and valgus in one patient. And six (18.7\%) patients with screws had a significant difference in their carrying angles,
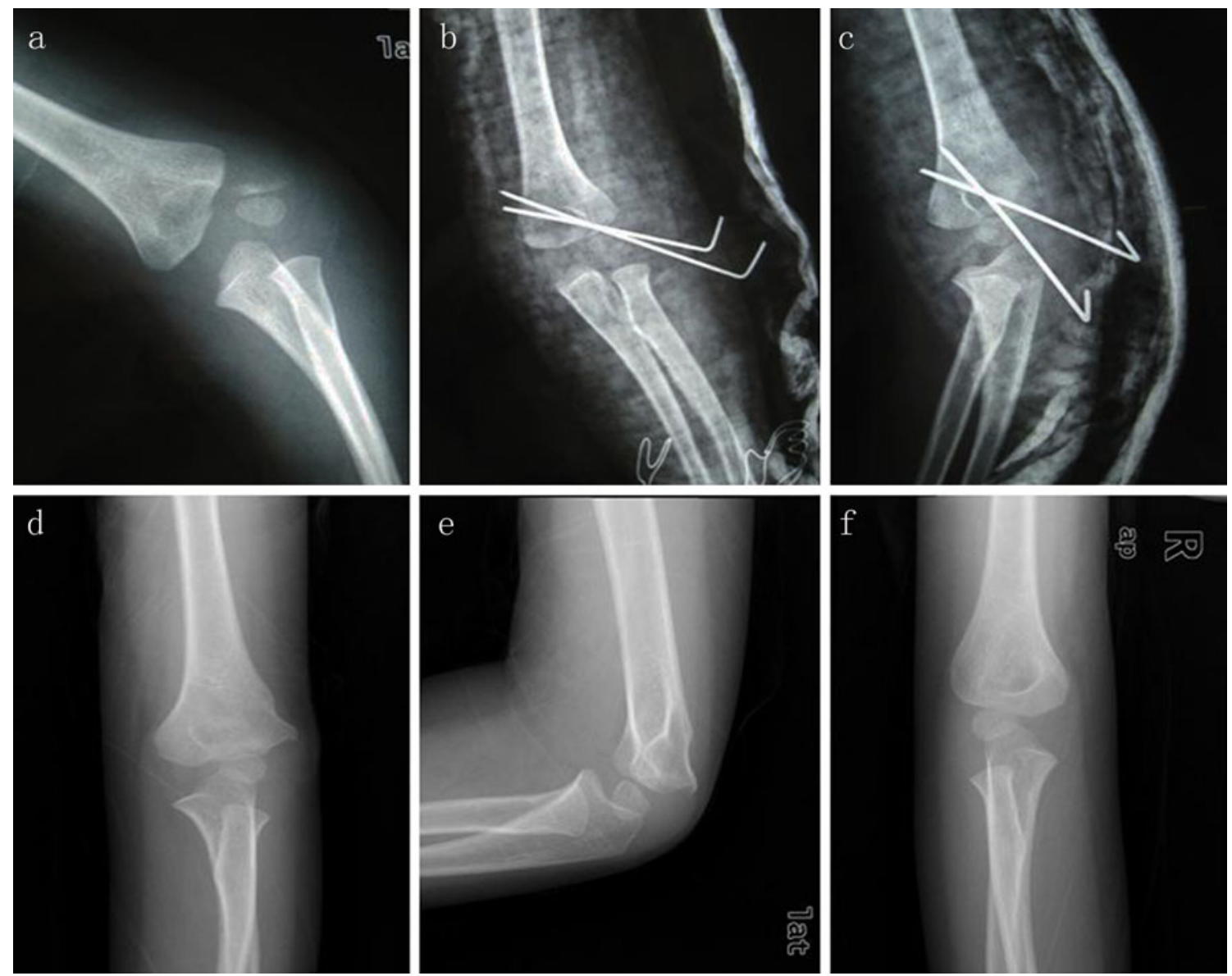

Fig. 2 Radiographs showed a displaced lateral humeral condyle fracture in the left elbow (a). The fracture was fixed with two smooth K-wires (b and $\mathbf{c}$ ). The follow-up radiographs (d and $\mathbf{e}$ ) showed the union of fracture with lateral prominence compared with the other side of elbow (f) 

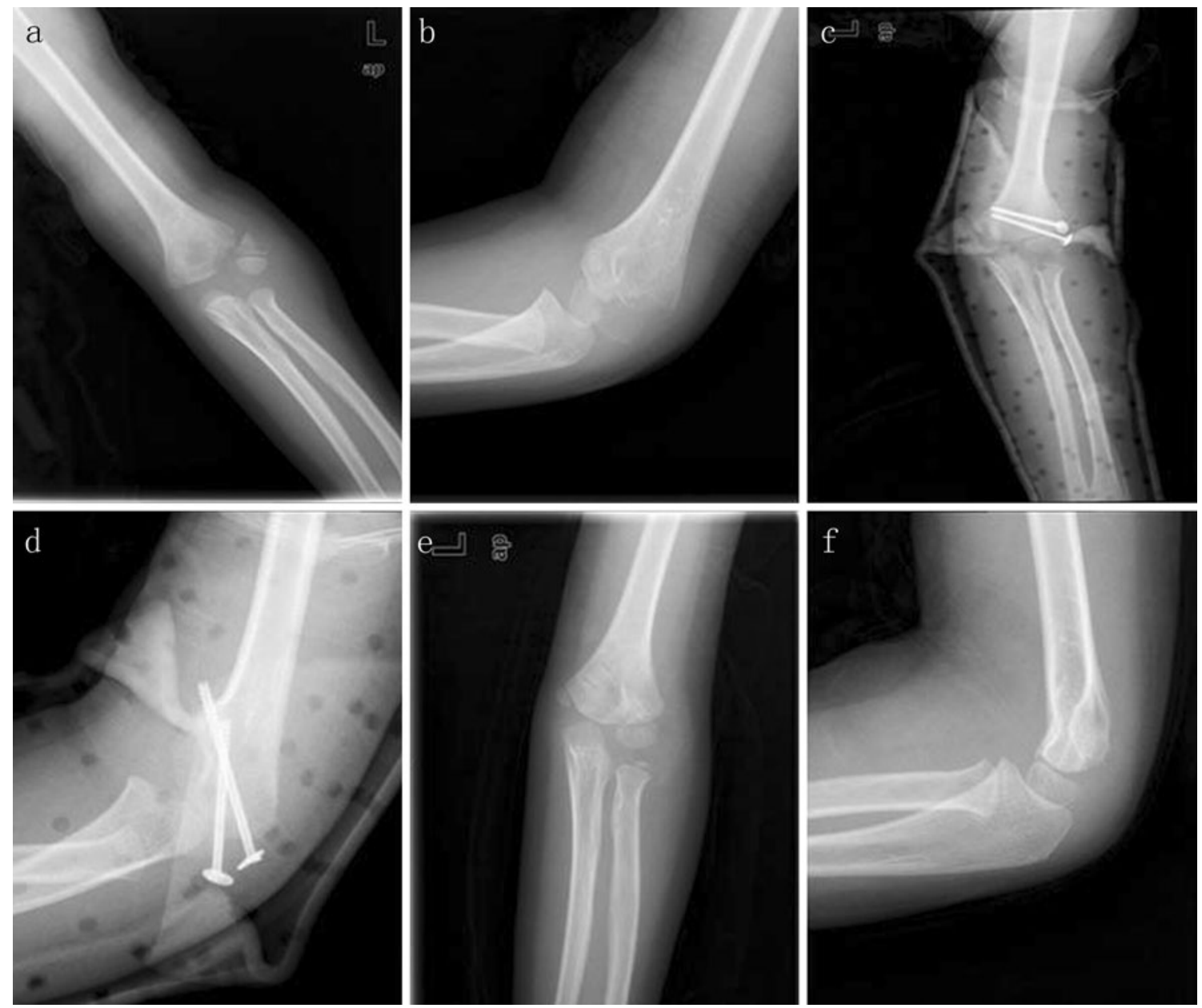

Fig. 3 A displaced and rotated lateral humeral condyle fracture in a 6-year-old boy (a and $\mathbf{b})$. The fracture was fixed with two cannulated screws and external stabilisation brace (c and $\mathbf{d})$. At the last follow-up, the radiographs showed good bone healing of fracture (e and $\mathbf{f}$ )

with relative cubitus varus in two patients and valgus in four patients. Among four patients with cubitus valgus, three were stabilised with screws through the ossific nucleus of capitellum. None required corrective osteotomy. Nine (30\%) patients with K-wires and two (6.3\%) patients with screws had a lack of $10^{\circ}$ of extension of the elbow compared with the other side. There was a statistically significant difference between the two groups in the lack of $10^{\circ}$ of extension of the elbow $(P<$ $0.05)$.

\section{Discussion}

There is a general agreement that the displaced lateral condyle fracture needs to be treated by open reduction and internal fixation. And internal fixation with $\mathrm{K}$-wires or cannulated screws has been reported to stabilise the displaced fractures [10-14]. In our study, we included all displaced fractures (over $2 \mathrm{~mm}$ ) treated by open reduction and smooth $\mathrm{K}$-wires or cannulated screw fixation. According to the criteria of Hardare et al. [19], there was no statistically significant difference in clinical outcome between the two groups. Radiographs showed that all fractures achieved union without avascular necrosis of the ossific nucleus.

Although K-wire was the common metallic internal fixation, a plaster splint or cast was required for a long period. Foster et al. [20] reported that elbow immobilisation should last for six to eight weeks after open reduction and K-wire fixation. Launay et al. [21] reported that patients received a long-arm splint or cast for an average 6.5 weeks (range four to ten weeks). Ayubi et al. [22] reported that a long upper-arm plaster cast should continue until wound healing in the fracture with K-wire fixation. Subsequently, an upper-arm plaster cast for three to four weeks postoperatively should be applied. In our study, an above-elbow plaster splint was used to support fractures with $\mathrm{K}$-wires for five or six weeks compared to fractures fixed with screws, followed by a brace for one to two weeks. The screws provided more continuous stabilisation for fracture than K-wires [12]. Patients could do exercises earlier and have better elbow function. There was a statistically significant difference between the two groups in the lack of $10^{\circ}$ of extension of the elbow. 
The K-wires left protruding outside the skin may increase the possibility of skin infection and the loosening of K-wires [7, 14, 18]. Weiss et al. [23] reported that $3.8 \%$ of patients developed skin infection around $\mathrm{K}$-wires and were treated with oral antibiotics. In our study, skin infection around the $\mathrm{K}$-wires occurred in $16.7 \%$ of patients, while no patients developed skin infection over cannulated screws. This indicated that more infection in patients occurred with percutaneous $\mathrm{K}$-wires than screws. Therefore, it is suggested that skin care should be done to prevent skin infection. Oral antibiotics and wound care should be given in treatment for infection. Although no infection occurred in the screws group, an additional operation was essential to remove screws with increased cost and length of stay.

Hardacre et al. [19] reported that K-wire fixation carried the risk of wire migration. In our group, seven K-wires in four fractures were found to be loose when K-wires did not pass through the cortex of the opposite side. We recommend that the double cortex fixation of smooth $\mathrm{K}$-wires is necessary if the K-wire is used. In addition, more than two Kwires fixation may also promote the stabilisation of fracture and prevent migration of K-wires. However, Launay et al. [21] found that the use of three K-wires could result in more loss of motion and a higher incidence of lateral spur than two K-wires. In our study, there were 11 patients with lateral prominence occurring in six patients with two $\mathrm{K}$-wires and in five patients with three $\mathrm{K}$-wires. We suggest that screws or more than two $\mathrm{K}$-wires can be used if the fracture is not stabilised sufficiently after two K-wires.

Lateral prominence was a common complication of the lateral condyle fractures. Thomas et al. [10] reported that $40 \%$ of patients with $\mathrm{K}$-wires had an obvious prominence over lateral humeral condyle, and the excessive bone formation beneath the osteoperiosteal flap may lead to lateral prominence. In our group, lateral prominence occurred in $36.7 \%$ of patients with K-wires and $12.5 \%$ of patients with screws. The difference in lateral prominence of the two groups was statistically significant. We thought the relatively low stabilisation of fracture with K-wires may cause more bone callus and the screws may promote the stabilisation of the fracture and reduce the probability of lateral prominence. Alternatively, the lateral prominence may be due to the wide dissection of periosteum and the formation of bone callus. So the periosteum in the metaphyseal fragment should be protected and wide dissection avoided.

Among four patients with cubitus valgus, three were stabilised with screws through the ossific nucleus of the capitellum. Although no avascular necrosis of ossific nucleus occurred, the results of cubitus valgus indicated that screws through the ossific nucleus may have a negative effect on growth plate in the lateral humeral condyle. Saraf et al. [17] advised that it was not possible to use screw fixation in the majority of fractures due to the risks of damage to the physis. But we suggest that screws can be used if there is enough space in the metaphyseal fragment. If not, $\mathrm{K}$-wire is still the first choice for the fracture.

\section{Conclusion}

This study has demonstrated several conclusions in treatment for lateral humeral condyle fractures with $\mathrm{K}$-wires or screws. First, the displaced fractures (displacement over $2 \mathrm{~mm}$ ) can be treated successfully by open reduction and $\mathrm{K}$-wires or screw fixation with excellent results. K-wires or screws should be removed when there is evidence of fracture union in follow-up radiographs. Second, K-wires can pass through the ossific nucleus of capitulum without damaging it. The percutaneous K-wires have the advantage of easy removal at the price of increasing the possibility of skin infection and $\mathrm{K}$-wire loosening. Last, although screws may have a negative effect on ossific nucleus of capitellum, and a second operation is needed for screw removal, they could reduce the possibility of lateral prominence and promote the union of fracture by continuously stabilising the displacement of fracture.

Conflict of interest The authors declare that they have no conflict of interest.

\section{References}

1. Marzo JM, d'Amato C, Strong M, Gillespie R (1990) Usefulness and accuracy of arthrography in management of lateral humeral condyle fractures in children. J Pediatr Orthop 10(3):317-321

2. Jakob R, Fowles JV, Rang M, Kassab MT (1975) Observations concerning fractures of the lateral humeral condyle in children. J Bone Joint Surg Br 57(4):430-436

3. Bast SC, Hoffer MM, Aval S (1998) Nonoperative treatment for minimally and nondisplaced lateral humeral condyle fractures in children. J Pediatr Orthop 18(4):448-450

4. Sharma H, Sibinski M, Sherlock DA (2009) Outcome of lateral humeral condylar mass fractures in children associated with elbow dislocation or olecranon fracture. Int Orthop 33(2):509-514. doi:10.1007/s00264-007-0463-1

5. Ersan O, Gonen E, Arik A, Dasar U, Ates Y (2009) Treatment of supracondylar fractures of the humerus in children through an anterior approach is a safe and effective method. Int Orthop 33 (5):1371-1375. doi:10.1007/s00264-008-0668-y

6. Skak SV, Olsen SD, Smaabrekke A (2001) Deformity after fracture of the lateral humeral condyle in children. J Pediatr Orthop B 10 (2):142-152

7. Toh S, Tsubo K, Nishikawa S, Inoue S, Nakamura R, Harata $S$ (2002) Long-standing nonunion of fractures of the lateral humeral condyle. J Bone Joint Surg Am 84-A(4):593-598

8. Hasler CC, von Laer L (2001) Prevention of growth disturbances after fractures of the lateral humeral condyle in children. J Pediatr Orthop B 10(2):123-130

9. Marcheix PS, Vacquerie V, Longis B, Peyrou P, Fourcade L, Moulies D (2011) Distal humerus lateral condyle fracture in children: when is 
the conservative treatment a valid option? Orthop Traumatol Surg Res 97(3):304-307. doi:10.1016/j.otsr.2010.10.007

10. Thomas DP, Howard AW, Cole WG, Hedden DM (2001) Three weeks of Kirschner wire fixation for displaced lateral condylar fractures of the humerus in children. J Pediatr Orthop 21(5):565-569

11. Cardona JI, Riddle E, Kumar SJ (2002) Displaced fractures of the lateral humeral condyle: criteria for implant removal. J Pediatr Orthop 22(2):194-197

12. Sharma JC, Arora A, Mathur NC, Gupta SP, Biyani A, Mathur R (1995) Lateral condylar fractures of the humerus in children: fixation with partially threaded $4.0-\mathrm{mm}$ AO cancellous screws. J Trauma 39(6):1129-1133

13. Baharuddin M, Sharaf I (2001) Screw osteosynthesis in the treatment of fracture lateral humeral condyle in children. Med J Malaysia 56(Suppl D):45-47

14. Conner AN, Smith MG (1970) Displaced fractures of the lateral humeral condyle in children. J Bone Joint Surg Br 52(3):460-464

15. Sulaiman AR, Munajat I, Mohd EF (2011) A modified surgical technique for neglected fracture of lateral humeral condyle in children. J Pediatr Orthop B 20 (6):366-371. doi:10.1097/ BPB.0b013e32834920df

16. Chou PH, Feng CK, Chiu FY, Chen TH (2008) Isometric measurement of wrist-extensor power following surgical treatment of displaced lateral condylar fracture of the humerus in children. Int Orthop 32(5):679-684. doi:10.1007/s00264-007-0380-3

17. Saraf SK, Khare GN (2011) Late presentation of fractures of the lateral condyle of the humerus in children. Indian J Orthop 45 (1):39-44. doi:10.4103/0019-5413.67119

18. Milch H (1964) Fractures and fracture dislocations of the humeral condyles. J Trauma 4:592-607

19. Hardacre JA, Nahigian SH, Froimson AI, Brown JE (1971) Fractures of the lateral condyle of the humerus in children. J Bone Joint Surg Am 53(6):1083-1095

20. Foster DE, Sullivan JA, Gross RH (1985) Lateral humeral condylar fractures in children. J Pediatr Orthop 5(1):16-22

21. Launay F, Leet AI, Jacopin S, Jouve JL, Bollini G, Sponseller PD (2004) Lateral humeral condyle fractures in children: a comparison of two approaches to treatment. J Pediatr Orthop 24(4):385-391

22. Ayubi N, Mayr JM, Sesia S, Kubiak R (2010) [Treatment of lateral humeral condyle fractures in children]. Oper Orthop Traumatol 22 (1):81-91. (in German) doi:10.1007/s00064-010-3006-2

23. Weiss JM, Graves S, Yang S, Mendelsohn E, Kay RM, Skaggs DL (2009) A new classification system predictive of complications in surgically treated pediatric humeral lateral condyle fractures. J Pediatr Orthop 29(6):602-605. doi:10.1097/BPO.0b013e3181b2842c 